\title{
Impact of intervening learning on resurgence in humans with Autism Spectrum Disorders
}

\author{
Phil Reed • Charlotte Clark
}

Published online: 29 December 2010

(C) Psychonomic Society, Inc. 20102010

\begin{abstract}
In the present study, we investigated the degree to which responding would resurge in children diagnosed with Autism Spectrum Disorders (ASD) following an intervening training period comprising different schedules of reinforcement. Twenty-four children of the ages 7-15, with a diagnosis of an ASD, were taught a play a sequence on a variable ratio(VR) 3 schedule of reinforcement, during a 30-min session. The play sequence was then extinguished before the participants were taught a second play sequence, using a VR-4 schedule for $30 \mathrm{~min}$, a VR-4 schedule for $60 \mathrm{~min}$, or a VR-2 schedule for $30 \mathrm{~min}$. A 5-min extinction session was then conducted to determine the impact that the intervening schedules had on the resurgence of the original behavior. The original sequence resurged to a greater extent for Group VR-4 $30 \mathrm{~min}$ than it did for the other two groups. The results provide evidence that the length of time between initial training and testing is not a prime determinant of the level of resurgence, but that the amount of conditioning may play a stronger role: The greater the number of reinforcers received, the smaller the resurgence effect.
\end{abstract}

\section{Keywords Resurgence $\cdot$ Humans $\cdot$ Autism}

Resurgence may be defined as the reappearance of previously observed behavior patterns that have not recently been observed in the behavioral repertoire. Although there are variations in the experimental procedures adopted for the study of resurgence, typically, an initial behavior is established (Response 1), and then this behavior is then extinguished while a new response (Response 2) is taught. This training continues until the behavioral repertoire contains

\section{P. Reed $(\square) \cdot$ C. Clark}

Department of Psychology, Swansea University,

Singleton Park,

Swansea, Wales SA2 8PP, UK

e-mail: p.reed@swansea.ac.uk
Response 2, but not Response 1. Finally, Response 2 is extinguished, and evidence concerning the re-emergence (resurgence) of Response 1 is examined. The results obtained from such studies show strong evidence that past learning will resurge into current behavior, even when it is not directly reinforced (Cleland, Foster, \& Temple 2000; Doughty, da Silva, \& Lattal 2007; Leitenberg, Rawson, \& Mulick 1975; Lieving \& Lattal, 2003; Reed \& Morgan, 2007).

Although resurgence effects have been studied most extensively in nonhumans (e.g., Cleland et al., 2000; Leitenberg et al., 1975; Lieving \& Lattal, 2003; Reed \& Morgan, 2007), the emergence of past patterns of behavior into the current repertoire has been taken to have implications for many clinical problems (see Bouton, 2002). Perhaps as a consequence of this applied importance, there are an increasing number of recent experimental demonstrations of resurgence effects in human participants (see Bruzek, Thompson, \& Peters 2009; Doughty, Cash, Finch, Holloway, \& Wallington 2010; Mechner, Hyten, Field, \& Madden 1997; Lieving, Hagopian, Long, \& O'Connor 2004; Wilson \& Hayes, 1996). For example, it has been suggested that resurgence plays a role in the emergence of severe challenging behavior (e.g., Lieving et al., 2004), although there have been few demonstrations with participants prone to show such behaviors - for example those with Autism Spectrum Disorders (ASD; but see Lieving et al., 2004; Volkert, Lerman, Call, \& Trosclair-Lasserre 2009), who also represent an important group of participants for behavior analytic research (see Lovaas, 1987; Osborne, McHugh, Saunders, \& Reed 2008; Reed, 2009).

The resurgence effect has been the subject of considerable theoretical speculation (e.g., Bouton \& Swartzentruber, 1991; Epstein, 1983; Rawson, Leitenberg, Mulick, \& Lefebvre 1977) and investigation with regard to the factors that produce the return of previously established behavioral 
patterns into the current repertoire (e.g., Doughty et al., 2007; Lieving \& Lattal, 2003; Shahan \& Chase, 2002). The present research focuses on the latter aspect of the exploration of resurgence. These influences can be divided, broadly, into three classes of factors: (a) those that have an impact on the strength and form of the original learning (Response 1); (b) those that have an impact on the strength and form of the intervening response (Response 2); and (c) those that affect the similarity, and associative strengths, of the test and training context.

The effect of factors associated with the strength and form of Response 1 on resurgence is relatively well understood. For example, factors that are associated with greater resurgence include the strength of the initial conditioning of Response 1 (Bruzek et al., 2009; Doughty et al., 2010). The form of the original response has also been shown to have an impact on subsequent resurgence (Doughty et al., 2007; Reed \& Morgan, 2007). In terms of the training-test context factors, the greater the similarity between the training and test contexts (Bouton, 2002), and the lower the rate of reinforcement in the test context relative to the training context (Lieving \& Lattal, 2003; Mechner et al., 1997), the greater will be the levels of resurgence.

However, the influence of the factors that are related to the interpolated period affect resurgence (i.e., the extinction of Response 1, and the conditioning of Response 2) have produced less consensus. Much work with nonhumans has suggested that the level of extinction of Response 1 is important to the level of resurgence of this response (Cleland et al., 2000; Leitenberg et al., 1975; but see Lieving \& Lattal, 2003). Leitenberg et al. conducted a series of studies focused on the extent to which resurgence is dependent on the degree of extinction that the initial response (Response 1) actually receives. Their results showed that when a new response (Response 2) was reinforced during the extinction of the original response (Response 1), higher rates of reinforcement for Response 2 produced a higher degree of resurgence for Response 1 when Response 2 was subsequently extinguished. The data presented by Leitenberg et al. suggest that this effect was probably due to the fact that there was a smaller chance of Response 1 contacting the extinction contingency (because of the potential for superstitious reinforcement in conditions with higher rates of reinforcement for Response 2); therefore, resurgence of Response 1 was more likely. Similarly, Cleland et al. altered the number of sessions during which Response 1 was exposed to extinction. Their results showed resurgence to be inversely related to the amount of exposure to extinction for Response 1: When Response 1 was weakened through large amounts of exposure to extinction contingencies prior to the reinforcement of Response 2, it was less likely to resurge during the extinction of Response 2.
However, other research suggests that resurgence is independent of the levels of extinction experienced by Response 1 . For example, Lieving and Lattal (2003) found that the level of resurgence did not depend on the period of reinforcement of Response 2 prior to the final extinction phase, and that it did not depend on the number of sessions of extinction. Additionally problematic to the interpretation of these effects is that such studies often confound the reduction in strength of Response 1 through its extinction, with the increase in strength of Response 2 (i.e., they reinforce Response 2 simultaneously with extinguishing Response 1 , which is often available for the participants to emit during conditioning of Response 2). Similarly, the level of extinction of Response 1 is often confounded with the passage of time from the learning of Response 1 to the test period (i.e., during the conditioning of Response 2, Response 1 can potentially be emitted and extinguished, meaning that there is both an increased amount of extinction and an increased period of time since conditioning of Response 1). These factors make it unclear as to whether it is the extinction of Response 1 (Leitenberg et al., 1975), the reinforcement of Response 2 (Lieving \& Lattal, 2003), or the passage of time since the conditioning of Response 1 (e.g., Mechner \& Jones, 2001; but see Doughty et al., 2010) that is critical in having an impact on resurgence.

Given the uncertainty about the impact of these factors on subsequent resurgence effects, the purpose of the present study was twofold: first, to examine resurgence with children diagnosed with ASD, about which there are few, if any, studies. Second, we wanted to examine the impact of the length and level of reinforcement of Response 2 on resurgence of Response 1. The resurgence procedure used in this experiment was developed to incorporate three phases, and was based on an integration of the procedures described for humans by Bruzak et al. (2009), and for pigeons by Lieving and Lattal (2003). In Phase 1, a response was taught to children with ASD, and was then extinguished. In Phase 2, the children were taught to emit a second response, without the opportunity to emit Response 1. The training of Response 2 was manipulated in terms of the length of exposure to this contingency, one group receiving 30-min exposure, and another receiving 60-min exposure. A third group received 30-min exposure to the contingency, but with a similar number of reinforcements as experienced in the 60-min exposure group (i.e., they had a richer training schedule). In a final test phase for resurgence, participants were allowed the opportunity to emit both Response 1 and Response 2 in extinction. In none of the groups could there be differential extinction of Response 1, as in no case were the participants able to emit Responsel during the interpolated learning of Response 2 . Thus, any effects would be the result of training of Response 2, which was the focus of the present investigation. 
These three groups were used to untangle the potential effects of length of exposure to Response 2 training from the number of reinforcements delivered. If resurgence were to be affected by the length of time since the initial training of Response 1, then the groups with 30-min interpolated training should show greater resurgence than the group with 60-min interpolated training. Although, if the present findings are consistent with those in previous literature (e.g., Bruzak et al., 2009; Lieving \& Lattal, 2003), this factor should not have an impact on resurgence performance. However, if the level of interpolated training were critical, then the group experiencing the leaner and shorter interpolated training (VR-4 $30 \mathrm{~min}$ ) might be expected to show greater levels of resurgence than the other two groups (VR-4 $60 \mathrm{~min}$ and VR-2 $30 \mathrm{~min}$ ).

\section{Method}

\section{Participants}

Twenty-four participants all had a diagnosis of Autism provided by a specialist pediatrician who was independent of this study. The diagnosis had been made in accordance with the DSM-IV criteria for Autism. The severity of the autism was assessed with the Autism Behavior Checklist $(\mathrm{ABC})$, which showed that the mean total $\mathrm{ABC}$ scores for the sample were $67.9( \pm 20.4)$, indicating a mild level of ASD for these participants. All of the participants could imitate, and respond to, verbal instructions. The age of participants ranged from 7 to 15 years old, and there were 20 males and four females. All of the participants attended the Jigsaw CABAS ${ }^{\circledR}$ School (Surrey, U.K.), which is an independent day school for children with ASD. Consent for participation in the study was granted by the parents or guardians of the participants. The participants were randomly divided into three groups.

\section{Apparatus}

The apparatus included five toys, which were as follows: "Turtles," "Play Doh," "Fuzzy Felt," "K'NEX," and "Mr. Potato Head." The participants' behavior emitted to these toys was taken as the responses for the purposes of this study. A response was taken to occur if a particular specified sequence of behavior related to a particular toy was emitted in total (see below for a description). This response sequence took a variety of times to emit across the participants, but generally required between 2 and $3 \mathrm{~s}$.

Sweets were used as reinforcement. These were the sweets that were typically used for each individual participant during their educational training, and included crisps and jelly tots. The type of sweet to be used for a particular individual was determined by asking the tutors delivering the applied behavior analysis program which sweet was preferred by a particular individual. Data sheets, pens, and timers were used to record the sessions.

\section{Procedure}

The study took place in the Jigsaw CABAS School. During all phases of the study, the participant sat at a desk next to the experimenter in a corner of their usual classroom. Each participant went through all phases of the study consecutively on the same day.

Preference assessment To determine the toys that would be played with as Response 1 and Response 2, a preference assessment test that was identical to that described by DeLeon and Iwata (1996) was conducted. In this test, the five toys were presented to the participant in a straight line, about $5 \mathrm{~cm}$ apart from one another, on the table at which the participant was sitting. The experimenter instructed the participant to select one toy. When an item was chosen from the array, it was then unavailable during the rest of the choice session. A selection response was recorded when the participant made physical contact with one of the presented items. After selection, the participant received $30 \mathrm{~s}$ in which to play with the selected toy. The left-to-right sequence of the remaining items was altered randomly. The second trial then followed in the same manner, and so on. The session continued until all of the toys had been selected, or until no toys were selected within $30 \mathrm{~s}$ from the beginning of the trial. If no toys were selected within $30 \mathrm{~s}$, the session ended, and all of the remaining items were recorded as not selected. Each participant was exposed to five consecutive sessions of the preference assessment. The two toys that had the lowest percentage score for being selected were chosen for each participant to be used in the experiment (randomly assigned as the toy for Response 1 and Response 2 ). This produced a wide variety of different combinations of toys for the participants.

Baseline A baseline session of 5 min was conducted for each participant in order to identify the sequence of play skills with the toy that were in the repertoire. Sessions were conducted in a quiet room, away from the classroom, in which the two least preferred toys were placed in front of the participant in a random order. The play sequences emitted by the participants were recorded. That is, the participant had free access to the toy, and the behaviors and the sequence in which they were emitted were recorded. A sequence was deemed to have been completed when the participant put down or moved away from the toy. Play sequences not emitted by any participant were then determined for each toy; these previously nonemitted play 
sequences were used as the precise response sequences to be taught in the present study.

The Fuzzy Felt sequence was adding black hair to the face, two pink circles as eyes, a green pupil to one eye, a dark pink circle to the other eye, two pink ears, a red nose, red lips, white teeth, and a blue bow.

For Play Doe, the sequence was to put the Play Doe in the cactus, push the cactus down on the set, roll the other Play Doe into a snake shape, and place the snake on the set.

For Mr. Potato Head, the sequence involved putting the feet on the potato, putting the spider coat on the potato, adding the Spiderman eyes, adding both arms, and then adding the spiderweb to the arm.

For the K'NEX, participants had to put a pink stick into a purple triangle, add the pink circle to the pink stick, put a second pink stick into the back of an eye piece, put the eye in the middle of the pink circle, add the two pieces of hair to the pink circle, and, finally, add a crown between the hair to the pink circle.

For the Turtles toy, the sequence was to line up the black monster, line up the purple monster, line up the green monster, squeeze the turtle's feet to knock over the black monster, squeeze the turtle's feet to knock over the purple monster, and squeeze the turtle's feet to knock over the green monster.

Phase 1 During Phase 1, the participants were taught the particular response sequence appropriate to the toy that served as their Response 1. The target sequence initially was modeled to the participant. Participants' independent correct responses were reinforced by a sweet edible and vocal praise. Corrections for incorrect responses were given by either vocally instructing the participants, or modeling the correct response. The participant received no reinforcement for corrected responses. Once the participants had completed the sequence, they were instructed to carry on, so that they would continue emitting the target sequence for the duration of the phase. The toy was not removed from the participant at any point during this phase.

All groups received reinforcement according to a VR-3 schedule in Phase 1. During this phase, only the toy associated with Response 1 was available to the participant (i.e., no reinforcement or extinction for other sequences was possible). Training lasted for $30 \mathrm{~min}$, then this response was placed into extinction, in which the participant could emit the trained response to the toy, but with no reinforcement. Extinction was taken to be complete when the participant did not emit the taught play sequence for 10 consecutive 5-s intervals.

Phase 2 Following the extinction of Response 1, one group of participants was taught the new response (Response 2) according to a VR-4 schedule, and this Phase 2 training continued for $30 \mathrm{~min}$. A second group of participants was taught Response 2 according to a VR-2 schedule, and this training lasted for $30 \mathrm{~min}$. The final group was taught according to a VR-4 schedule, but their training lasted $60 \mathrm{~min}$. During this phase, only the toy associated with Response 2 was available to the participant (i.e., no reinforcement or extinction for other sequences was possible).

Resurgence test A final 5-min test session was conduct in which each participant was given the two toys that they had been trained with in Phase 1 and Phase 2, and participants' behavior was recorded.

Interrater observation A second observer was used for interobserver agreement (IOA) in order to provide interobserver reliability (IOR), and was used to independently collect data. The IOA was conducted on $30 \%$ of the participants, for all of the sessions during all of the phases. IOA was calculated by dividing the number of agreements regarding the behavioral sequence emitted by the total number of behavioral sequences emitted and multiplying by 100. In all cases, the IOA was $100 \%$ in all phases.

\section{Results}

The group rates of response in the first and last 3-min block of training in Phase 1 (training Response 1) and in Phase 2 (training Response 2), can be seen in Fig. 1. In Phase 1, there were $10 \times 3$ min blocks for all groups. In Phase 2, there were $10 \times 3$ min blocks for Group VR-4 $30 \mathrm{~min}$ and VR-2 $30 \mathrm{~min}$, but $20 \times 3$ min blocks for Group VR-4 $60 \mathrm{~min}$. An inspection of these data reveals that all groups came to be engaged with the toy in more intervals by the

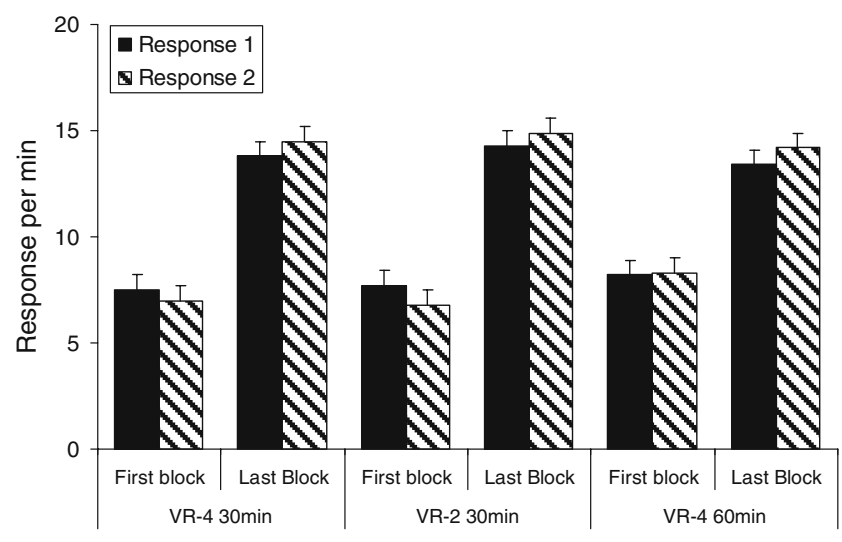

Fig. 1 The mean group rates of response (error bars $=$ standard deviations) during the first, and last, 3-min block of training, for each phase, for each group 
end of training in each phase than at the beginning of each phase. By the end of each phase, the participants were almost continually engaged with the toy.

A three-factor mixed-model ANOVA with group as a between-subjects factor and response (Response 1 vs. Response 2) and block (first vs. last) as within-subjects factors was conducted on these data. This and all subsequent analyses used a $p<.05$ significance criterion. This analysis revealed a statistically significant main effect of block, $F(1,21)=194.13, p<.001$, but no other main effects or interactions were statistically significant.

Figure 2 shows the group mean number of 5-s intervals taken to reach the extinction criteria for Response 1 (10 successive 5-s intervals without a response to the toy) for the three groups. All groups extinguished responding to Response 1 in about 150-180 s, and an ANOVA revealed no statistically significant difference between the groups in time taken to reach this criterion, $F<1$.

The top panel of Fig. 3 displays the group mean rate for Response 1, in each minute of extinction in the test session. Response 1 increased for the VR-4 30-min group over the first few minutes, and also increased, albeit to a lesser extent, for Group VR-4 60 min. There was little responding noted for Group VR-2 $30 \mathrm{~min}$. By the end of the extinction test, there were few responses emitted by any group. A twofactor mixed-model ANOVA (Group x Interval) was conducted on these data and revealed significant main effects of group, $F(1,21)=32.34, p<.001$, and interval, $F(4,84)=21.99, p<.001$, and a significant interaction between the factors, $F(8,84)=6.04, p<.001$. Simple effect analyses revealed a significant difference between the groups on Intervals 1 to 4 , inclusive [smallest $F(2,84)=$ $18.11, p<.001]$. Subsequent Tukey's HSD tests conducted on those intervals for which a significant simple effect of group had been observed revealed that for Interval 1, there was a significant difference between Group VR-4 $30 \mathrm{~min}$ and VR-2 $30 \mathrm{~min}$. For Interval 2, all pairwise comparisons

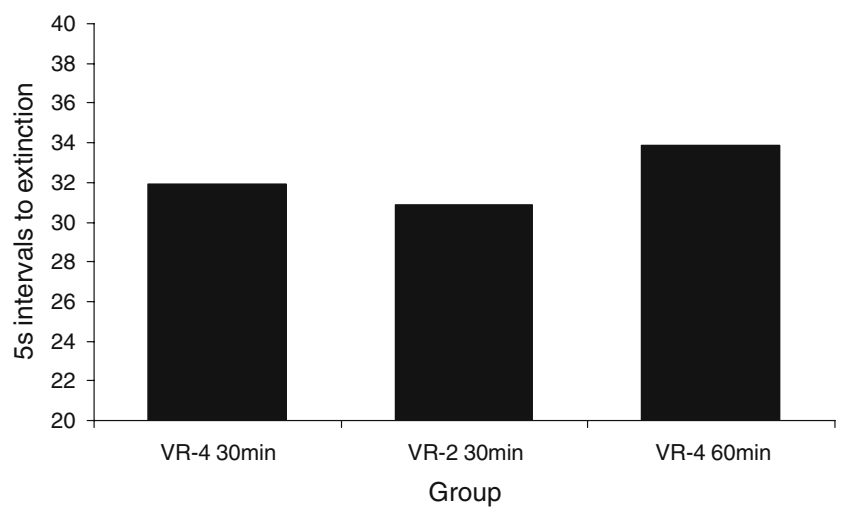

Fig. 2 The group mean number of 5-s intervals until extinction (ten 5-s intervals without a response) for the Response 1 for each group
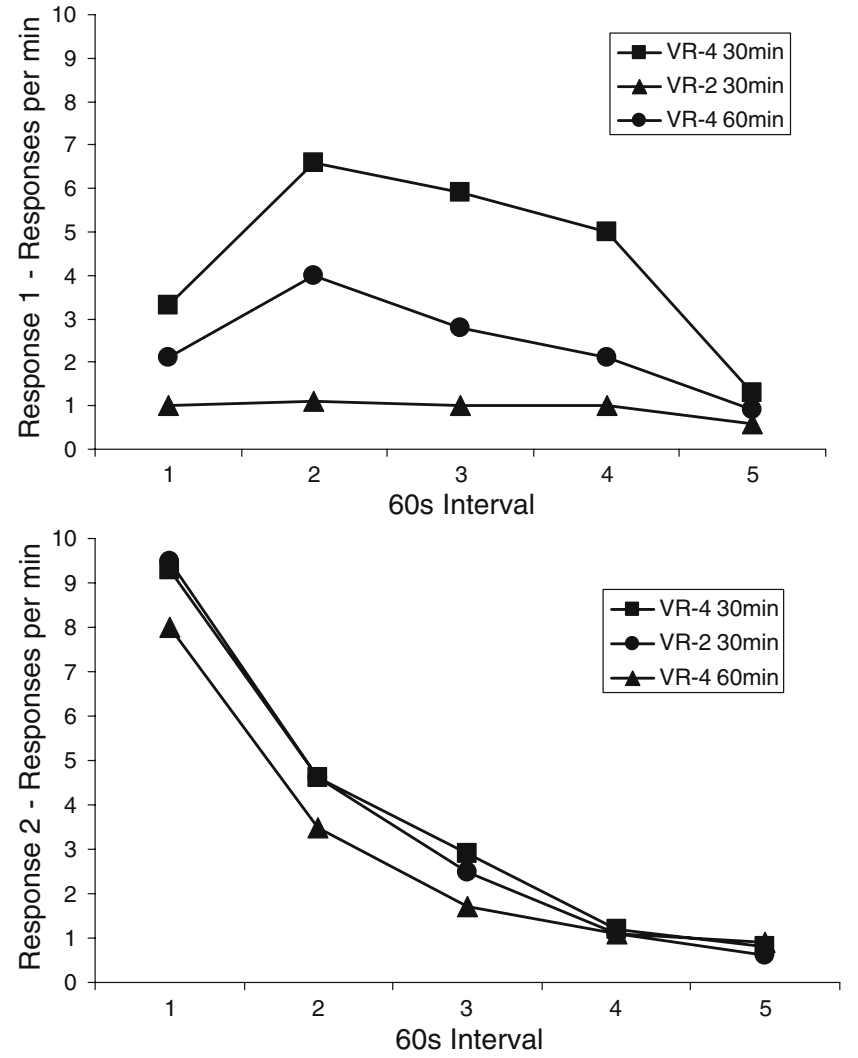

Fig. 3 Group mean rate of Response 1 during each minute of extinction test for each group (top panel). Group mean rate of Response 2 during each minute of extinction test for each group (bottom panel)

were significant; on Intervals 3 and 4, there were differences between Group VR-4 $30 \mathrm{~min}$ and each of the other groups.

The bottom panel of Fig. 3 shows the group mean rate of Response 2, in each minute of extinction. These data show a decrease in responding over the course of extinction, which was consistent across all three groups. A two-factor mixedmodel ANOVA (Group x Interval) was conducted on these data and revealed a significant main effect of interval, $F(4$, $84)=203.22, p<.001$, but neither the main effect of group nor the interaction were significant, $p \mathrm{~s}>.20$.

The mean (standard deviation) rate of reinforcement earned for Response 2 during Phase 2 for each group was: Group VR-4 $30 \mathrm{~min}=2.9( \pm 0.1)$; Group VR-2 $30 \mathrm{~min}=$ $5.9( \pm 1.8)$; and Group VR-4 $60 \mathrm{~min}=3.1( \pm 0.6)$. An ANOVA conducted on these data revealed a significant difference between the groups, $F(2,21)=189.26, p<.001$, with Tukey's HSD tests showing that the only significant pairwise differences were between Group VR-2 30 min and each of the other two groups.

The total numbers of reinforcers earned for the three groups during Phase 2 were: Group VR-4 $30 \mathrm{~min}=86.8$ $( \pm 3.1)$; Group VR-2 $30 \mathrm{~min}=179.8( \pm 5.5)$; and Group VR- 
$460 \min =187.7( \pm 3.6)$. An ANOVA conducted on these data revealed a significant difference between the groups, $F(2,21)=180.99, p<.001$, with Tukey's HSD tests showing that the only significant pairwise differences were between Group VR-4 30 min and each of the other two groups.

\section{Discussion}

The aim of the present study was to identify the degree to which an originally learned response would show resurgence in human participants with ASD, and the extent to which the learning of the interpolated response in Phase 2 had an impact on resurgence. The results demonstrated that resurgence was clearly noted in a group trained on an interpolated response on a VR-4 schedule for $30 \mathrm{~min}$. This group displayed stronger resurgence of Response 1 than did a group trained on the same schedule for $60 \mathrm{~min}$, and one trained on a VR-2 schedule for $30 \mathrm{~min}$. Although the former of these two groups displayed greater numerical levels of resurgence than the latter, this difference was only statistically reliable on one out of five sessions. These findings suggest that the length of time between initial training and testing is not a prime determinant of the level of resurgence; Groups experiencing the same number of reinforcers for Response 2 exhibited the same levels of resurgence as one another, despite having different lengths of interpolated training. Rather, it appears that the amount of conditioning may play a stronger role (the greater the number of reinforcers received, the smaller the resurgence effect). Although, speculatively, the slight difference between the VR-4 60-min and VR-2 30-min groups may also suggest that massed interpolated training reduces resurgence more effectively than does spaced training.

Note that there was no difference in the rates of extinction of Response 2 across the final (resurgence test) phase. This suggests that the results in the test relating to Response 1 are not differentially affected by the levels of extinction responding for Response 2 . However, there are a number of caveats that should be considered when interpreting these data, especially with regard to a number of additional control groups that may well be useful to employ in future research. First, it should be noted that there was no control included to assess the level of spontaneous recovery of responding during extinction. The absence of this control does not interfere with the ability to make inferences regarding the group differences, which was the primary purpose of the present study, but it does mean that care should be taken when interpreting the absolute levels of resurgence. Second, only two responses (toys) were available to the participants during the resurgence test. If additional responses (toys) also were presented, then the level of resurgence of Response 1 could have been compared with the occurrence of a novel response to determine whether the interpolation of Response 2 after initial training with Response 1 had the assumed effects on resurgence. The fact that there were group differences and that there was minimal activity in the VR-2 30-min groups does suggest some differential impact of Response 2 training, but further investigation of this issue is warranted.

A further caveat, and area for potential further exploration, concerns the apparent relationship between the level of reinforcement given to Response 2 in the interpolated training (Phase 2) and the subsequent resurgence of Response 1 during test. If a lower level of reinforcement of Response 2 produces greater resurgence, then it might be suggested that no reinforcement would produce the greatest resurgence. Although a procedure in which reinforcement was not provided for an alternative response may invoke different mechanisms than those seen in resurgence, this is an issue that deserves some comment and further exploration. That this was the pattern of results observed in the present study might suggest that some mechanism such as spontaneous recovery was responsible for the present effect (although the fact that length of time between initial extinction and test did not have an impact on the recovery of responding suggests this explanation is unlikely). However, future studies could address this by including groups in which Response 2 was never presented, or was presented but never reinforced.

In addition, it may be that the manner in which the alternative response (Response 2) receives reinforcement could be a critical variable to explore. Leitenberg et al. (1975) presented data that suggest that rate of reinforcement is directly correlated with resurgence, which is in contrast with the data from the present study, but also that length of training (and presumably numbers of reinforcers) are inversely correlated with resurgence effects - a finding that is in line with those from the present report. Separating these two variables and exploring their impact on resurgence more clearly may well be of interest.

These results add to the literature that demonstrates that resurgence, although widely reported in nonhuman subjects (e.g., Cleland et al., 2000; Doughty et al., 2007; Lieving \& Lattal, 2003; Reed \& Morgan, 2007; see Shahan \& Chase, 2002), is readily found with humans. These demonstrations have included work using similar responses to those studied in nonhumans (e.g., button presses), as well as those involving more complex response patterns (Bruzak et al., 2009; Mechner \& Jones, 2001) and higher order responses (Doughty et al., 2010; Wilson \& Hayes, 1996). It is still unclear whether precisely the same factors influence the demonstration of resurgence across species, and much greater documentation and convergence of procedures will be necessary to establish this issue. Nevertheless, the 
present results strengthen the documentation of this effect in humans and extend this to a population with ASD.

The present results are broadly in line with suggestions that it is not only the level of extinction of Response 1 that is critical in determining the level of subsequent resurgence. Leitenberg et al. (1975; see also Cleland et al., 2000) reported data that suggest that this factor may be important; however, given the potential confounds in those studies that were discussed in the introduction, and given the pattern of the present results, it can be concluded that, even when levels of extinction of Response 1 are equated across groups, resurgence is affected by additional factors. In the present study, it was very unlikely that any participants emitted Response 1 during the training of Response 2, because the toy to which Response 1 was emitted was not present, meaning that Response 1 could not have been extinguished (Cleland et al., 2000) or reinforced superstitiously (Leitenberg et al., 1975). To this extent, the present findings support those of Lieving and Lattal (2003; see also Doughty et al., 2007) and suggest that factors independent of the levels of extinction of Response 1 also have an impact on resurgence.

The present findings also suggest that prime among the additional factors that may affect resurgence is the strength of conditioning to Response 2. It has been established that levels of conditioning of Response 1 are implicated in resurgence effects (Bruzak et al., 2009), and that the typography of Response 2 is important (Doughty et al., 2007); however, the present data suggest that the amount of reinforcement also has an impact on resurgence; the greater the numbers of reinforcers experienced for Response 2, the smaller the resurgence effect. In the present study, both groups that had greater numbers of reinforcers for Response 2 (VR-4 $60 \mathrm{~min}$ and VR-2 $30 \mathrm{~min}$ ) displayed less resurgence than did the third group (VR-4 $30 \mathrm{~min}$ ). There was also a suggestion that the delivery of these reinforcers in a massed rather than spaced manner tended to lower the levels of resurgence, in that Group VR-2 30 min displayed less resurgence than did Group VR-4 $60 \mathrm{~min}$. However, this difference was relatively transitory in the present report and potentially requires further study. It is interesting to note that massed extinction trials led to less relapse in drug abuse (Corty \& Coon, 2000), which may be related to the present results.

There was little evidence in the present study that length of time since the original training, per se, had an impact on resurgence. It was the case that the VR-4 group who received 60-min training with Response 2 displayed less responding than the VR-4 group that had 30-min training with Response 2, but, as was discussed previously, this is more likely to be due to the numbers of reinforcement experienced in the 60-min group, given that similar low levels of resurgence were seen in the VR-2 group that experienced only a 30-min Response 2 training period. This supports previous findings (e.g., Bruzak et al., 2009;
Lieving \& Lattal, 2003) and implies that any "time-based" effects are more likely because of the strength of interpolated conditioning of intervening responses.

There are a number of additional theoretical accounts related to the occurrence of resurgence that should be mentioned, although discriminating between these accounts was not the primary purpose of this study. The responseprevention hypothesis (Rawson et al., 1977), the extinctioninduced resurgence hypothesis (Epstein, 1983), and contextual conditioning (Bouton \& Swartzentruber, 1991) have all been suggested as explanations for resurgence. The present data to not readily speak to the latter of these two views, but they do suggest that the response-prevention hypothesis cannot provide a comprehensive account of resurgence. Rawson et al. suggested that under some conditions, the operative contingencies (e.g., punishment) serve to prevent the target response from contacting the extinction contingency. Once these contingencies are withdrawn, the target response is available to be emitted again. In resurgence studies, when the target response is extinguished alongside the reinforcement of the interpolated response, this could also occur. However, in this case, the target response was extinguished prior to the reinforcement of the interpolated response, making the response prevention hypothesis unable to account for the present results.

Some comment is needed on the use of children with ASD in the present study. The primary purpose of selecting such a group was to extend the range of populations over which resurgence phenomena can be demonstrated, and the present results show that resurgence is observed in children with ASD. This is important, because several authors (e.g., Lieving et al., 2004) have suggested that resurgence is a key factor in the emergence of severe behavioral problems. The extension of basic research to this group would, therefore, appear to strengthen the assumptions made in this applied work.

In summary, the results of the present study have suggested that resurgence effects of broadly similar types as occur in nonhumans are also noted in human subjects, in this instance, children with ASD. The data also suggest a role of the strength of the interpolated response in determining levels of resurgence, over and above any impact of the directly conditioned strength of the initially trained response.

Acknowledgments Thanks are due to the participants in the research, and to their families, as well as to the Jigsaw school for their cooperation, and to Lisa A. Osborne for her support and comments on this research.

\section{References}

Bouton, M. E. (2002). Context, ambiguity, and unlearning: sources of relapse after behavioral extinction. Biological Psychiatry, 52, 976-986. 
Bouton, M. E., \& Swartzentruber, D. (1991). Sources of relapse after extinction in Pavlovian and instrumental learning. Clinical Psychology Review, 11, 123-140.

Bruzek, L., Thompson, R. H., \& Peters, L. C. (2009). Resurgence of infant caregiving responses. Journal of the Experimental Analysis of Behavior, 92, 327-343.

Cleland, B. S., Foster, T. M., \& Temple, W. (2000). Resurgence: the role of extinction. Behavioural Processes, 52, 117-129.

Corty, E. W., \& Coon, B. (2000). The extinction of naturally occurring conditioned reactions in psychoactive substance users: analog studies. Addictive Behaviors, 20, 605-618.

DeLeon, I. G., \& Iwata, B. A. (1996). Evaluation of a multiplestimulus presentation format for assessing reinforcer preferences. Journal of Applied Behavior Analysis, 29, 519-533.

Doughty, A. H., Cash, J. D., Finch, E. A., Holloway, C., \& Wallington, L. K. (2010). Effects of training history on resurgence in humans. Behavioural Processes, 83, 340-343.

Doughty, A. H., da Silva, S. P., \& Lattal, K. A. (2007). Differential resurgence and response elimination. Behavioural Processes, 75, $115-128$.

Epstein, R. (1983). Resurgence of previously reinforced behavior during extinction. Behaviour Analysis Letters, 3, 391-397.

Leitenberg, H., Rawson, R. A., \& Mulick, J. A. (1975). Extinction and reinforcement of alternative behavior. Journal of Comparative and Physiological Psychology, 88, 640-652.

Lieving, G. A., Hagopian, L. P., Long, E. S., \& O'Connor, J. (2004). Response-class hierarchies and resurgence of severe problem behavior. Psychological Record, 54, 621-634.

Lieving, G. A., \& Lattal, K. A. (2003). Recency, repeatability, and reinforcer retrenchment: an experimental analysis of resurgence. Journal of the Experimental Analysis of Behavior, 80, 217-233.
Lovaas, O. I. (1987). Behavioral treatment and normal educational functioning in young autistic children. Journal of Consulting and Clinical Psychology, 55, 3-9.

Mechner, F., Hyten, C., Field, D. P., \& Madden, G. J. (1997). Using revealed operants to study the structure and properties of human operant behavior. Psychological Record, 47, 45-68.

Mechner, F., \& Jones, L. (2001). Number of prior repetitions of operants, and resurgence. Retrieved from http://mechnerfoundation.org/ pdf_downloads/number_of_repetitions.pdf

Osborne, L. A., McHugh, L., Saunders, J., \& Reed, P. (2008). Parenting stress reduces the effectiveness of early teaching interventions for Autistic Spectrum Conditions. Journal of Autism and Developmental Disorders, 38, 1092-1103.

Rawson, R. A., Leitenberg, H., Mulick, J. A., \& Lefebvre, M. F. (1977). Recovery of extinction responding in rats following discontinuation of reinforcement of alternative behavior: a test of two explanations. Animal Learning \& Behavior, 5, 415-420.

Reed, P. (2009). Behavioural theories and interventions for autism. New York: Nova Science Publishers.

Reed, P., \& Morgan, T. A. (2007). Resurgence of behavior during extinction depends on previous rate of response. Learning \& Behavior, 35, 106-114.

Shahan, T. A., \& Chase, P. N. (2002). Novelty, stimulus control, and operant variability. Behavior Analyst, 25, 175-190.

Volkert, V. M., Lerman, D. C., Call, N. A., \& Trosclair-Lasserre, N. (2009). An evaluation of resurgence during treatment with functional communication training. Journal of Applied Behavior Analysis, 42, 145-160.

Wilson, K. G., \& Hayes, S. C. (1996). Resurgence of derived stimulus relations. Journal of the Experimental Analysis of Behavior, 66, $267-281$. 